Corresponding author: michele.fedel@unitn.it Tel: 00390461282403

Fax: 00390461281977

\title{
Waterborne acrylic paint system based on nanoceria for corrosion
} protection of steel

\author{
L.G Ecco $^{1}$, M. Fedel ${ }^{1}$, F. Deflorian ${ }^{1}$ \\ Jacob Becker ${ }^{2}$, Bo Brummerstedt Iversen ${ }^{2}$, Aref Mamakhel ${ }^{2}$ \\ ${ }^{1}$ Department of Industrial Engineering, University of Trento, Trento - Italy \\ 2 Center for Materials Crystallography, Department of Chemistry and iNANO, Aarhus \\ University, Langelandsgade 140, DK-8000, Aarhus, Denmark
}

\begin{abstract}
The corrosion protection efficiency of steel painted with waterborne acrylic coatings loaded with diverse concentration of cerium oxide nanoparticles are presented in this paper. The investigation was carried out by means of Electrochemical Impedance Spectroscopy (EIS) and the salt spray test. EIS has been conducted at two experimental conditions: the intact coating condition as well as in presence a purposely created macroscopic defect. EIS observations from the intact coatings evidenced the higher protection for the paint systems containing the ceria nanoparticles compared to that without the nanoceria, e.g. higher magnitudes for the charge transfer resistance after certain period of exposure to sodium chloride solution. In addition to that, from the evolution of the double layer capacitance on the defected coatings condition, reduced delamination rates were perceived for those containing 0.5 and $1.0 \mathrm{wt}$. \% of nanoceria. Similarly, after exposure to the salt spray test a reduced delaminated area was suggested for $0.5,1.0$ and $2.0 \mathrm{wt}$ \% compared to that without nanoceria. Generally, a beneficial contribution of nanoceria into an acrylic waterborne paint system on the corrosive protection of steel was found and the optimal concentration of nanoceria associated to higher corrosion protection was verified for the paint system containing 1.0 wt. \%.
\end{abstract}

Key words: Nanoceria, EIS, Salt spray test, Corrosion protection, Steel 


\section{Introduction}

Carbon steel is extensively used in many fields of applications. It is relatively inexpensive and a wide range of mechanical properties can be obtained by simply alloying elements or thermal treatments. On the other hand, corrosion protection is required since carbon steels have low corrosion resistance [1].

Among all existing technologies to prolong the lifetime of steel, the use of organic coatings is a cost effective method. Corrosion protection provided by solventborne organic coatings is well-known and have been widely used in several fields of application, however environmental issues, mainly the emission of volatile organic compounds (VOC's) to the atmosphere, are attributed to the use of solventborne organic coatings. Besides, the use of inhibiting compounds, such as hexavalent chromium $\left(\mathrm{Cr}^{6+}\right)$, is recognized to provide sufficient corrosion protection for steel, nevertheless $\mathrm{Cr}^{6+}$ compounds are considered to have hazardous consequences for the environment and human health [2].

In order to overcome the above scenario, European environmental protection directives have limited the use of solvent based organic coatings [3] and restricted the use of $\mathrm{Cr}^{6+}$ [4]. Consequently, corrosion science and industry have been boosted towards the development of more environmentally friendly and viable alternatives for replacing $\mathrm{Cr}^{6+}$ as well as reduce the use of solvent based organic coatings.

On the lookout of alternatives to reduce the emissions of VOC's, waterborne coatings emerged as environmentally friendly candidate to replace the traditional solvent-based coatings [5]. In the beginning, the main drawbacks attributed to waterborne coatings were intrinsically associated to the use of water as a dispersive medium for the coating formulation, for instance the flash rusting concern. As well, waterborne coatings were presumed to have lower chemical resistances and consequently shorter service life compared to solventborne [6]. However, latest progresses made on the chemistry of waterborne coatings, e.g. development of water compatible functionalized groups for enhanced chemical resistance [7], along with a better comprehension on the film formation mechanisms [8] have yielded them comparable to solventborne coatings. In 
addition, waterborne coatings have gained attention due to their economic viability $[9,10]$.

Simultaneously to the development of waterborne coatings and seeking alternatives to replace the hazardous compounds, nanoscale cerium oxide appears as a possible candidate. $\mathrm{CeO}_{2}$ denotes the stable stoichiometric form of bulk cerium oxide and it shows all of the cerium in the $\mathrm{Ce}(\mathrm{IV})$ state. However, the $\mathrm{CeO}_{2}$ particles when reduced into nanoscale (nanoceria) show physical and chemical properties which differ from the bulk form [11]. Nanoceria is currently used in many industrial applications, such as catalysts, semiconductor polishing agents and full additives [12,13]. Likewise, they have been studied for many other applications such as biomedical application [14] and UV absorbers $[15,16]$.

Similarly, corrosion science has recently increased attention in the application of nanoceria for corrosion protection of steel. Recalling previous studies on the use of cerium oxide nanoparticles and the corrosion of mild steel, eventual inhibition effects have been perceived. EIS observations suggested an electrochemical response of a blocking electrode of steel immersed into the chloride solutions containing nanoceria [17]. Succeeding, the presence of $1.0 \mathrm{wt}$. \% of nanoceria into a solventborne alkyd coating improved anticorrosive protection properties of the steel substrate. The effectiveness of the particles on the inside of the coatings has been perceived from the evolution of the impedance at low frequency range and in the charge transfer resistance [18]. Particularly in reference [18], the efficiency of nanoceria on the corrosion protection was verified in a single layer organic coating with thickness $10 \mu \mathrm{m}$ containing the nanoparticles however, due to the relatively low thickness, the coatings underwent to a quick degradation rate.

With the purpose to overcome this condition and go deeper into this subject, the effect of the nanoceria has been assessed into a more genuine waterborne organic coating paint system. This study includes an optimization of the concentration of nanoceria in the primer's layer of a two layer paint system for the corrosion protection of mild steel. For that, nanoceria produced via homogeneous precipitation method were incorporated at diverse concentration levels into the primer of a two layer paint system and coated 
onto low carbon steel. Prior to the incorporation into the coatings, the physical properties of the nanoceria were studied by means of powder X-ray diffraction, UV-Vis spectroscopy and completed with high resolution TEM. Once incorporated into the coatings, the protective properties of the paint systems have been studied by means of EIS and salt spray test. For that, two different test conditions were used to conduct the investigation: the intact and scratched conditions.

\section{Experimental}

\subsection{Synthesis of nanoceria}

The nanoceria particles were synthesized via precipitation of $\mathrm{CeCl}_{3} \cdot 6 \mathrm{H}_{2} \mathrm{O}$ with $\mathrm{NH}_{4} \mathrm{OH}$ in presence of citric acid. All the chemicals were purchased from Sigma Aldrich and used as received. An aqueous solution of $0.5 \mathrm{M} \mathrm{CeCl}_{3}$ and $0.5 \mathrm{M}$ citric acid was prepared under mechanical stirring. Cerium chloride was selected as the $\mathrm{Ce}^{+3}$ ions precursor whereas citric acid acted as steric stabilizer of the nanoparticles in order to obtain a stable colloidal dispersion. Next, a $24.5 \%$ aqueous $\mathrm{NH}_{4} \mathrm{OH}$ was slowly added into the cerium ions containing solution until $\mathrm{pH}$ was stabilized between 9-10. Ammonia acted as precipitating ligand, it promoted reaction and precipitation of cerium hydroxide species due to its low solubility in the alkaline medium [19]. Later, nucleation and growth of $\mathrm{CeO}_{2}$ from the hydroxide species was favored in the alkaline environment in presence of oxygen [20]. The mixture was heated to $90{ }^{\circ} \mathrm{C}$ and held at this temperature for $24 \mathrm{~h}$ and then was cooled to room temperature $\left(\sim 23{ }^{\circ} \mathrm{C}\right)$. The resulting $\mathrm{CeO}_{2}$ dispersion was precipitated by adding excess ethanol, which enabled separation by centrifugation. The yellow "cake" of particular $\mathrm{CeO}_{2}$ was rinsed three times with demineralized water and ethanol in order to remove chloride ions. Finally, it was redispersed in pure water with a loading of $10 \mathrm{wt} . \%$ and $\mathrm{pH}$ of water solution containing nanoceria adjusted to approximately 8 with $\mathrm{NH}_{4} \mathrm{OH}$. The water based dispersion was stable for several months. 


\subsection{Waterborne acrylic paint}

Following the process of synthesis, the nanoceria were incorporated into a waterborne coating at diverse levels of concentration: 0.5, 1.0, 2.0 and 3.0 wt. \%. A clear waterborne acrylic binder was used. The polymer resin was supplied by Arkema Coating Resins (Verneuil en Halatte, France) and Table 1 reports the main properties of the binder. The values reported in Table 1 were provided by the manufacturer. The dispersion of the nanoparticles into the binder and their homogenization were done via mechanical stirring. Following, the painted steel samples were prepared and mild steel panels from Q-Panel $(75 \times 150 \mathrm{~mm})$ with arithmetic roughness of $0.15 \mu \mathrm{m}$ were used as substrate. Prior to the coatings applications, the surface of steel was degreased with ultra-sonication in presence of acetone. No further surface adhesion treatment was implemented before the application of the coatings.

The waterborne acrylic binder containing the nanoceria was the first layer over the steel panels and from now on it would be mentioned as the primer layer. On top of the primary layer, the same waterborne acrylic binder containing $3.0 \mathrm{wt}$ \% of bentonite (Laviothix P100 from Laviosa Chimica Mineraria S.p.A. - Italy) was applied. The thicknesses of each were found near 10 and $25 \mu \mathrm{m}$ for the primer and the top layer, respectively. Figure 1 schematizes the two layer paint system. The addition of the top layer containing the bentonite intended to enhance the physical barrier property of the paint system. In this way, while testing, the ingress of water throughout the primer layer was sufficiently lowered and the concentration of nanoceria was ensured to be the parameter of investigation.

The coatings applications were done with a Bar Coater. Table 2 summarizes the main characteristics of the studied paint systems. Before testing, the coating thicknesses for the all the panels were controlled with Phynix equipment, model Surfix FN according to the standard ASTM D1186-93 [23]. 


\subsection{Characterization}

Characterization of the as-prepared $\mathrm{CeO}_{2}$ nanoparticles was done via powder $\mathrm{X}$-ray diffraction collected with $2 \%$ min and $2 \theta=20-80$ degrees, step in 2 theta 0.01 degree in Bragg-Brentano geometry (Rigaku SmartLab, $\mathrm{Cu}-\mathrm{Ka}_{1}$ radiation). Transmission Electron Microscopy, (FEI Talos F220A) operated at 200 kV. Samples were dispersed and ultrasonicated in absolute ethanol and deposited on a holey carbon copper grid. UV-VIS Spectroscopy was recorded in the range of 200-700 nm with optical diffuse reflectance measurements performed at room temperature $\left(\sim 23{ }^{\circ} \mathrm{C}\right)$ with $\mathrm{BaSO}_{4}$ as the reference sample (Shimazu UV-3101 PC).

The protective properties of the paint systems were studied by Electrochemical Impedance Spectroscopy (EIS). The single-sine EIS measurements were obtained at the open circuit potential (OCP) using a potentiostat and frequency response analyzer (FRA) equipment (Autolab Metrohm PGstat 302N). The measurements were carried out with the classical three electrodes arrangement; a reference electrode with internal solution $\mathrm{Ag}|\mathrm{AgCl}|(+0.205 \mathrm{~V}$ vs $\mathrm{SHE})$ and a platinum wire as counter electrode. The frequency was scanned from $10^{5}$ to $10^{-2} \mathrm{~Hz}$ and the testing area, i.e. coating area in contact with the electrolyte was $10 \mathrm{~cm}^{2}$. All the measurements have been conducted at room temperature. Both, a waiting time of 5 minutes alongside a potential stabilization of $\mathrm{dE} / \mathrm{dt}$ lower than $1 \mathrm{mV} / \mathrm{s}$ were respected before the acquisition of the spectra. EIS investigation has been conducted at two different experimental conditions; intact coatings as well as in presence of an intentional macroscopic defect $1.0 \mathrm{~cm}$ long, as shown in Table 3. The quantitative analysis, i.e., spectra's fitting using an equivalent electrical circuit was made using the software ZSimpWin 3.22.

The salt spray test was conducted in presence of an intentional defect. Exclusively for this test, the length of the intentional defect was equal to $2 \mathrm{~cm}$. The coated panels were inserted into the chamber according to the standard ASTM B117 [24]. All the EIS and salt spray outcomes presented and discussed below are representative of duplicated measurements. No significant variations were observed and the repeatability has been considered reliable. 


\section{Results and Discussion}

\subsection{Nanoceria}

The optical responses of the ceria nanoparticles analyzed by UV-Vis spectroscopy are shown in Figure 2. It can be seen a strong absorption peak bellow $300 \mathrm{~nm}$ in correspondence to the band gap energy $\left(E_{g}\right)$ of ca. $3.70 \mathrm{eV}$, as displayed in the picture inserted in Figure 2. The $\mathrm{E}_{\mathrm{g}}$ of the synthesized $\mathrm{CeO}_{2}$ samples has been estimated according the modified Kubelka-Munk method as demonstrated in Eq. 1, where $h$ is the Planck's constant, $v$ is the light frequency, $B$ is a constant, $F(R)=\left(1-R^{2}\right) / 2 R, R$ is the reflectance. [23].

$$
[\mathrm{F}(\mathrm{R}) \times h v]^{\mathrm{n}}=\mathrm{B}\left(h v-\mathrm{E}_{\mathrm{g}}\right)
$$

Eq.1

The expected absorption peak of the bulk $\mathrm{CeO}_{2}$ is near to $410 \mathrm{~nm}$ in correspondence to the band gap energy near $3.02 \mathrm{eV}$ [26]. Compared to bulk $\mathrm{CeO}_{2}$ the absorption peak was shifted towards shorter wavelength in correspondence to higher $E_{g}$ for the asprepared nanoceria. These are due to quantum-size confinement effect, i.e. more energy will be needed to be absorbed by the band gap of the material. Thus, these observations are indicative on the nanoscale of the particles [15].

The XRD patterns of the as-prepared nanoceria are shown in Figure 3. The obtained powder diffractogram revealed diffraction peaks corresponding to the (111), (200), (220) and (311) reflections. The peaks can be indexed as cubic fluorite phase of according to the Joint Committee on Powder Diffraction Standards (JCPDS) card number: 00-0340394. The $\mathrm{CeO}_{2}$ crystallite size was estimated by application of Scherrer equation (Eq. 2) was found near $2-3 \mathrm{~nm}$ :

$$
D_{\mathrm{XRD}}=0.94 \lambda / B \cos \theta
$$

Where $\lambda$ is the wavelength of $x$-rays, $B$ is the full width at half maximum (FWHM) of the $\mathrm{CeO}_{2}$ (111) peak and $\theta$ the peak position $(\theta \sim 14.28)$. Likewise, by means of high resolution TEM the nanoscale of $\mathrm{CeO}_{2}$ particles was demonstrated. Fig. 4 shows the particle size for a single crystal near 2-3 nm. 


\subsection{Waterborne paint systems based on ceria nanoparticles}

Figures 5 displays the EIS spectra of the intact paint systems at the beginning of the analysis and their evolution after 24 and $192 \mathrm{~h}$ of exposure to $0.1 \mathrm{M} \mathrm{NaCl}$ solution. At the beginning, the EIS spectra for all the paint systems were relatively similar. There was less than one order of magnitude difference on the values of the total impedance at $10^{-2} \mathrm{~Hz}\left(|\mathrm{Z}|_{0.01 \mathrm{~Hz}}\right)$. Values were found between $10^{8}$ and $10^{9} \mathrm{Ohm} . \mathrm{cm}^{2}$.

In the course of time differences started to be noticed between the samples. After $24 \mathrm{~h}$ the values of $|Z|_{0.01 \mathrm{~Hz}}$ of W.ACR-0.5 and W.ACR-1 were moved towards higher values. The magnitudes for these two paint systems were found near $10^{9} \mathrm{Ohm} . \mathrm{cm}^{2}$ whereas those for W.ACR-0, W.ACR-2 and W.ACR-3 were reduced. Besides, the phase angle spectra for all the paint systems revealed the presence of a second time constant in the frequency range of $10^{0}-10^{-2} \mathrm{~Hz}$, as an indicative that corrosion reactions or any electrochemical process at the steel/coating interface have begun.

After $192 \mathrm{~h}$, the EIS evolution indicated degradation process of W.ACR-0. There was a drop on the $|\mathrm{Z}|_{0.01 \mathrm{~Hz}}$ to $10^{7} \mathrm{Ohm} . \mathrm{cm}^{2}$ and the second time constant in the middle to low frequency range of the phase angle spectrum suggested corrosion reactions on the surface of steel. The W.ACR- 0.5 evolved with significant stability during this period of time. The W.ACR-1 showed a slight reduction on the magnitudes of $|Z|_{0.01 \mathrm{~Hz}}$, from $10^{9}$ to $10^{8} \mathrm{Ohm} . \mathrm{cm}^{2}$. Interesting was the the evolution of W.ACR-2 and W.ACR-3 from 24 to $192 \mathrm{~h}$, there was a shift towards the lower frequencies on their phase angle spectra accompanied by a minor increase in the magnitudes of $|\mathrm{Z}|_{0.01 \mathrm{~Hz}}$.

Figure 6 shows the evolution of $|Z|_{0.01 \mathrm{~Hz}}$ after approximately $1600 \mathrm{~h}$. The values of $|\mathrm{Z}|_{0.01 \mathrm{~Hz}}$ for W.ACR-0 were found stable near $10^{8} \mathrm{Ohm} . \mathrm{cm}^{2}$ during the first $48 \mathrm{~h}$ and started to reduce after that. At the end of the analysis they were found near $10^{5}$ Ohm. $\mathrm{cm}^{2}$ indicating the loss of protective property of this coating. In the cases of W.ACR-0.5 and W.ACR-1 the $|Z|_{0.01 \mathrm{~Hz}}$ evolved as follows: from the beginning until the $48^{\text {th }}$ hour the values were increased from $10^{8}$ to $10^{9} \mathrm{Ohm} . \mathrm{cm}^{2}$ and remained stable up to the $250^{\text {th }}$ hour. After, they were constantly reduced and verified near $10^{6} \mathrm{Ohm} . \mathrm{cm}^{2}$ at the 
end of the test. For W.ACR-2 and W.ACR-3, the initial values of $|Z|_{0.01 \mathrm{~Hz}}$ were detected near $10^{8} \mathrm{Ohm} . \mathrm{cm}^{2}$. Succeeding, after the $50^{\text {th }}$ hour there was a shift in the trend of $|\mathrm{Z}|_{0.01 \mathrm{~Hz}}$, the values started to move towards higher magnitudes and finally reduced again after $300 \mathrm{~h}$.

The values and the evolution $|Z|_{0.01 \mathrm{~Hz}}$ represent the physical contribution of both, the impedance attributed to coating layer itself together with the impedance associated to faradaic processes occurring at the interface coating layer/ steel [27]. Therefore, the evolution of $|Z|_{0.01 \mathrm{~Hz}}$ towards higher magnitudes for W.ACR-0.5 and W.ACR-1 in the first $24 \mathrm{~h}$ and similarly for W.ACR-2 and W.ACR-3 after certain period of exposure can be attributed either to barrier effect or to any electrochemical activity of nanoceria at the steel surface, according to [17]. In order to go deeper into this and to quantify the contribution of nanoceria, EIS data was modelled with the equivalent electrical circuits shown in Fig 7.

In the equivalent circuit shown in Figure $7(\mathrm{a}), \mathrm{R}_{\mathrm{e}}$ stands for the electrolyte resistance, $R_{P o}$ for the pore resistance attributed to the coating and $Q_{C c}$ for the constant phase element (CPE) of the coating, associated with the coating capacitance. In Figure 7 (b), the two additional electrical parameters $Q_{C d l}$ and $R_{c t}$ represent, respectively, the CPE associated to the double-layer capacitance and the charge transfer resistance. The use of CPEs instead of a pure capacitance, i.e. coating capacitance and double layer capacitance, was justified for a better fitting between experimental data and the electrical circuits [28].

Figure $8(\mathrm{a})$ shows the evolution of the pore resistance during the first $400 \mathrm{~h}$ of exposure. There was a reduction of approximately one order of magnitude, from $10^{8}$ to $10^{7} \mathrm{Ohm} . \mathrm{cm}^{2}$, in the first $24 \mathrm{~h}$ for all of the coatings. The drop of the $\mathrm{R}_{\mathrm{Po}_{\mathrm{o}}}$ values was due to the ingress of water and ions from the electrolyte into the coatings layer. Longer periods of exposure revealed differences between the samples. In the cases of W.ACR0, W.ACR-0.5 and W.ACR-1 there was a continuous reduction of the pore resistance yet explained by the ingress of water and ions. Differently, the evolution of $R_{p o}$ for W.ACR-2 and W.ACR-3 after 24 h, despite the water uptake, changed direction and 
started to move towards higher values. The maximum magnitude, near $10^{8} \mathrm{Ohm} . \mathrm{cm}^{2}$, was reached after $300 \mathrm{~h}$ and then the values started to decrease.

Next, the evolution of the charge transfer resistance of the paint systems as function of time is seen in Figure $8(b)$. The initial data for $R_{c t}$ and its evolution became plausible after the $9^{\text {th }}$ hour. Before that, no electrochemical reactions at the interface steel/ coating layer were detected. In the case of W.ACR-0 the values of $R_{\text {ct }}$ were initially found around $10^{9} \mathrm{Ohm} . \mathrm{cm}^{2}$, remained stable at these magnitudes up to $200 \mathrm{~h}$ then the values decreased. The values of $R_{c t}$ for W.ACR- 0.5 moved towards higher values from the beginning of the test until the $24^{\text {th }}$ hour and then were reduced. Similar, those for W.ACR-1 evolved in the direction of higher values throughout the first $96 \mathrm{~h}$ before being reduced.

Considering the changes on the $R_{p o}$ and $R_{c t}$ values for W.ACR-0.5 and W.ACR-1, the evolution of $|\mathrm{Z}|_{0.01 \mathrm{~Hz}}$ depicted for these paint systems in Fig 6 can now be interpreted as a consequence of partial reduction of faradaic processes occurring at the interface coating layer/ steel. Higher charge transfer resistances values denote a deceleration of the processes of transferring charges when the electrons reached the metal and, simultaneously, the metal ions diffused into the wet coating or electrolyte [27]. It is likely that the presence of 0.5 and $1.0 \mathrm{wt}$. \% of ceria nanoparticles in the primer layer of acrylic paint reduced the rates of the transferring charges and consequently the overall corrosion reactions were lowered.

In the case of W.ACR-2 $R_{c t}$ values continuously increased until the $200^{\text {th }}$ hour, there was an increase of one order of magnitude, from $10^{8}$ to $10^{9} \mathrm{Ohm} . \mathrm{cm}^{2}$ and after that started to decrease. W.ACR-3 showed stable values near $10^{8} \mathrm{Ohm} . \mathrm{cm}^{2}$ until the $300^{\text {th }}$ hour then started to reduce the magnitudes. From the observation of the $R_{c t}$ values along with the evolution of $R_{p o}$, it is likely that the contribution of of 2.0 and $3.0 \mathrm{wt}$. \% of nanoceria reflected upon the coating layer rather than at the interface coating layer/ steel. In other words, for these weight concentrations it seems that nanoceria enhanced mainly the physical barrier effect of the paint. 
Following, EIS has been conducted on the coatings in presence of a purposely created defect. The macroscopic defect intended to establish an active corrosion area around the defect. In this way, the diffusion of the electrolyte through the coating layer was less significant compared to the coating delamination on the scratchy zone during the tested period of $24 \mathrm{~h}$. As a consequence, the EIS spectra of the scratched coatings were modelled with the 2 time constant equivalent electrical circuit depicted in Figure 7(b). The influence of cerium oxide nanoparticles has been assessed from the evolution the CPE associated to the double-layer capacitance. Figure 9 shows the evolution in the course of $24 \mathrm{~h}$ for the double-layer capacitance after certain time $\left(\mathrm{Q}_{\mathrm{Cdl} t \mathrm{t} \mathrm{t}^{*}}\right)$ over the double layer capacitance at the beginning of the exposure $\left(Q_{C d l}=0\right)$ of the scratched coatings.

For W.ACR-0, an increase of the ratio $Q_{C d l} t=t^{*} / Q_{C d l} t=0$ from the beginning of the test until the $24^{\text {th }}$ hour is seen. The evolution of W.ACR-2 is comparable to the W.ACR-0 and the final values of $Q_{C d l} t=t^{*} / Q_{C d l} t=0$ were verified to W.ACR-3. On the other hand, in the cases of W.ACR-0.5 and W.ACR-1, the final values of the normalized double layer capacitance were approximately 4 times lower than those of the W.ACR-0.

The electrochemical parameter $Q_{C d l}$ is an indicative about the wet area underneath the paint [29]. In addition to that, the delamination rate of the coatings can be assessed by examining the evolution of the normalized double layer capacitance [30]. The proposed assessment as a rough estimation of the delaminated area from the scratchy zone towards the edges of the panels in the case of macroscopically defected coatings has been demonstrated elsewhere [31]. Therefore, if one considers the ratio $Q_{C d l} t=t^{*} / Q_{C d l}$ $\mathrm{t}=0$ as a parameter to assess the delamination rate that occurred from the scratchy zone towards the edges of the panel, the drop of about 4 times evidenced a remarkable decline of the delamination rate is promoted by the addition of 0.5 and $1.0 \mathrm{wt}$. \% of ceria nanoparticles. Possible explanations for W.ACR-2, W.ACR-3 and their higher rates of delamination are, the presence of aggregates in the primer's layer allowing the faster moving of the electrolyte underneath paint. Or else, the inhibition effect given by the nanoceria was limited to the optimal concentration of 0.5 and $1.0 \mathrm{wt}$. \%. 
Lastly, Figure 10 shows the macroscopic aspect of the scratched paint systems after 24 $\mathrm{h}$ of exposure to salt spray test. Remembering, the coatings application was done without any adhesion treatment on the steel surfaces. Looking at Figure 10, the samples showed blistering around the defect and the amount of blisters decreased in the order of W.ACR. 3 > W.ACR. 0 > W.ACR. 2 > W.ACR. $0.5>$ W.ACR.1.

On the whole, by means of UV-Vis spectroscopy, XRD diffraction and TEM, in Figs 2-4, the nanoscale of the synthesized cerium oxide particles have been confirmed and the presence of these cerium oxide nanoparticles in the primer layer of a waterborne acrylic paint system lowered the overall corrosion rates of steel and prolonged the corrosion protection efficiency of the paint systems. Significant findings have been observed for the coatings containing 0.5 and 1.0 wt. $\%$ of $\mathrm{CeO}_{2}$ nanoparticles. Particularly, EIS observations have shown an increasing trend on the values $|Z|_{0.01 \mathrm{~Hz}}$ during the initial period of immersion and the fitting of the spectra revealed an evolution towards higher values for the charge transfer resistance during concomitant period of time. These outcomes are suggestive that nanoceria seems to act electrochemically at the interface coating layer/ steel. It is likely that the typical corrosion reactions, or those that occurred for W.ACR-0 while testing, were suppressed in the cases of acrylic waterborne with 0.5 and 1.0 wt. \%. Furthermore, EIS and salt spray test operated on the defected coating condition revealed reduced delaminated areas for the coatings loaded with 0.5 and 1.0 wt. \% of nanoceria.

According to reference [17], the steel surface in contact with water based dispersion containing $\mathrm{CeO}_{2}$ nanoparticles underwent to significantly low corrosion rates while testing. The outcomes have suggested the passivation of steel surface and for these reasons the present study investigated the effectiveness of $\mathrm{CeO}_{2}$ nanoparticles when embedded inside an organic coating. The obtained results did not unequivocally discriminate the contribution of the $\mathrm{CeO}_{2}$ nanoparticles as additives/pigments which has led to a coating with higher barrier property from their contribution as corrosion inhibitor. However, it was observed that the presence of 0.5 and 1.0 wt. $\%$ of $\mathrm{CeO}_{2}$ inside the coatings, even if did not remarkably affect the barrier property (see Figure 8a), promoted an increase of the charge transfer resistance attributed to effects at the 
interface steel/coating layer. Considering the first $24 \mathrm{~h}$ of immersion all the coatings show approximately equivalent values of Rpo (as depicted in Figure 8a), meaning a comparable barrier property, while the samples containing 0.5 and 1.0 wt. $\%$ of $\mathrm{CeO}_{2}$ shows increased values of Rct (as depicted in Figure 8b).

These observations are in accordance with the findings reported in [17] where a stabilization of steel surface triggered by the presence of $\mathrm{CeO}_{2}$ has been observed. In addition, the delamination rate of the coatings containing 0.5 and $1.0 \mathrm{wt} \%$ of $\mathrm{CeO}_{2}$, demonstrated by EIS and salt spray test, cannot be related to an improvement of the barrier property caused by the presence of the nanoceria at these weight concentrations.

On the other hand, the outcomes observed for the coatings containing 2.0 and $3.0 \mathrm{wt}$ \% of $\mathrm{CeO}_{2}$ were different. $\mathrm{CeO}_{2}$ nanoparticles, at these weight concentrations, do not have proved effective to reduce delamination rate. The reason for such a different behavior in unclear to the authors. Nonetheless, it is possibly to mention about a partial agglomeration of the particles which hinder the inhibition effect. Moreover, it was observed that despite the ingress of water and ions, the evolutions of the impedance at low frequency range and pore resistance towards higher values occurred after $50 \mathrm{~h}$ of immersion. In this way, the addition of 2.0 and $3.0 \mathrm{wt}$. \% nanoceria particles seems to mainly enhanced the physical barrier effect of the coatings.

\section{Conclusion}

This paper reported the assessment by means of Electrochemical Impedance Spectroscopy and Salt Spray test on the corrosion protection efficiency of a waterborne acrylic paint containing nanoceria for protection of steel.

The cerium oxide nanoparticles were synthesized via the precipitation of cerium chloride in presence of citric acid. The nano scale of the particles, near to $2.0 \mathrm{~nm}$, has been confirmed by means of powder X-ray diffraction and Transmission Electron Microscopy. 
Generally, enhanced corrosion protection efficiency has been obtained due to the incorporation of nanoceria into the primer layer of waterborne acrylic paint. Whether the contribution to higher corrosion protection efficiency was inhibitive or physical depended on the weight concentration of the as synthesized nanoceria into the coating layer. From the results obtained in this work the contribution of nanoceria to higher corrosion protection efficiency has the optimal weight concentration of $1.0 \%$, the action of the nanoparticles were perceived at the interface coating layer/steel. When concentrations higher than that were added there was a shift from an inhibitive to a coating with higher physical barrier effect coating.

\section{Acknowledgments}

This work was supported by the SteelCoat Project (NMP-1910.1.2-2 Substitution of materials or components utilizing Green Nanotechnology. Project Number: 263262) funded by FP7 pro-gram of European Commission. The work was further supported by the Danish National Research Foundation (DNRF93) and by the Brazilian research foundation CNPq "Conselho Nacional de Desenvolvimento Científico e Tecnológico Brasil". 


\section{References}

[1] D.A. Jones, Principles and Prevention of Corrosion, $2^{\text {nd }}$ ed., Prentice-Hall Inc, 1996. ISBN 13: 9780133599930

[2] Z.W. Wicks, F.N. Jones, S.P. Pappas, Organic Coatings: science and technology, $2^{\text {nd }}$ ed.,1999. ISBN: 978-0-471-67475-7

[3] The European Commission, COUNCIL DIRECTIVE 1999/13/EC, Off. J. Eur. Union. 1998 (1992) 4580-4582.

[4] The European Commission, DIRECTIVE 2004/42/CE OF THE EUROPEAN PARLIAMENT AND OF THE COUNCIL, (2011).

[5] E. Potvin, L. Brossard, G. Larochelle, Corrosion protective performances of commercial low-VOC epoxy/urethane coatings on hot-rolled 1010 mild steel, Progress in Organic Coatings. 31 (1997) 363-373.

[6] A. Wegmann, Chemical resistance of waterborne epoxy/amine coatings, Progress in Organic Coatings. 32 (1997) 231-239.

[7] V. Duecoffre, W. Diener, C. Flosbach, W. Schubert, Emulsifiers with high chemical resistance: a key to high performance waterborne coatings, Progress in Organic Coatings. 34 (1998) 200-205.

[8] N.A. Swartz, T.L. Clare, Understanding the differences in film formation mechanisms of two comparable solvent based and water-borne coatings on bronze substrates by electrochemical impedance spectroscopy, Electrochimica Acta. 62 (2012) 199- 206.

[9] J. L Keddie, Film formation of latex, Materials Science and Engineering, 21 (1997) 101-170. 
[10] C.Y. Bai, X.Y. Zhang, J.B. Dai, W.H. Li, A new UV curable waterborne polyurethane: Effect of $\mathrm{CC}$ content on the film properties, Progress in Organic Coatings 55 (2006) 291-295.

[11] J.C. Clinton, Colloidal Cerium Oxide Nanoparticles: Synthesis and Characterization Techniques, Techniques. (2008).

[12] E.K. Goharshadi, S. Samiee, P. Nancarrow, Fabrication of cerium oxide nanoparticles: Characterization and optical properties, Journal of Colloid and Interface Science. 356 (2011) 473-480.

[13] M. Zawadzki, Preparation and characterization of ceria nanoparticles by microwave-assisted solvothermal process, Journal of Alloys and Compounds 454 (2008) 347-351.

[14] G. Renu, V.V.D. Rani, S V. Nair, K.R.V. Subramanian, V.K. Lakshmanan, Development of cerium oxide nanoparticles and its cytotoxicity in prostate cancer cells, Advanced Science Letters. 5 (2012) 5, 1-9.

[15] K. Anupriya, E. Vivek, B. Subramanian, Facile synthesis of ceria nanoparticles by precipitation route for UV blockers, Journal of Alloys and Compounds 590 (2014) 406410.

[16] S. Yabe, T. Sato, Cerium oxide for sunscreen cosmetics, Journal of Solid State Chemistry 171 (2003) 7-11.

[17] M. Fedel, A. Ahniyaz, L.G. Ecco, F. Deflorian, Electrochemical investigation of the inhibition effect of $\mathrm{CeO} 2$ nanoparticles on the corrosion of mild steel, Electrochimica Acta 131 (2014) 71-78.

[18] L.G. Ecco, M. Fedel, A. Ahniyaz, F. Deflorian, Influence of polyaniline and cerium oxide nanoparticles on the corrosion protection properties of alkyd coating, Progress in Organic Coatings. 77 (2014) 2031-2038. 
[19] Hayes S A., Pu Y.., Thomas J. O'K., Matthew J. O'Keefe, James O. S., The Phase Stability of Cerium Species in Aqueous Systems, Journal of Electrochemical Society. 149 (2002) C623.

[20] H. I. Chen, H. Y. Chang, Homogeneous precipitation of cerium dioxide nanoparticles in alcohol / water mixed solvents, Colloids and Surfaces A: Physicochem. Eng. Aspects 242 (2004) 61-69.

[21] ISO 3251 - Paints, varnishes and plastics: Determination of non-volatile matter content.

[22] ISO 2555 - Plastics - Resins in the liquid state or as emulsions or dispersions Determination of apparent viscosity by the Brookfield Test method.

[23] ASTM D1186-93 - Standard Test Methods for Nondestructive Measurement of Dry Film Thickness of Nonmagnetic Coatings Applied to a Ferrous Base.

[24] ASTM B117 - Standard Practice for Operating Salt Spray (Fog) Apparatus.

[25] R. López, R. Gómez, Band-gap energy estimation from diffuse reflectance measurements on sol-gel and commercial $\mathrm{TiO}_{2}$ : a comparative study, Journal of SolGel Science and Technology. 61 (2012) 1-7.

[26] N.K. Renuka, Structural characteristics of quantum-size ceria nano particles synthesized via simple ammonia precipitation, Journal of Alloys and Compounds 513 (2012) 230- 235.

[27] A. Amirudin, D. Thierry, Application of electrochemical impedance spectroscopy to study the degradation of polymer-coated metals, Progress in Organic Coatings. 26 (1995) 1-28.

[28] M. Musiani, M.E. Orazem, N. Pébère, B. Tribollet, V. Vivier, Determination of resistivity profiles in anti-corrosion coatings from constant-phase-element parameters, Progress in Organic Coatings 77 (2014) 2076-2083. 
[29] R. Hirayama, S. Hanuyama, Electrochemical impedance for degraded coated steel having pores, Corrosion. 47 (1991) 952-958.

[29] F. Deflorian, S. Rossi, L. Fedrizzi, P.L. Bonora, EIS study of organic coating on zinc surface pretreated with environmentally friendly products, Progress in Organic Coatings. 52 (2005) 271-279.

[30] F. Deflorian, S. Rossi, M. Fedel, G. Pilzer, L. Fedrizzi Characterisation of Silane Pretreatment for Organic Coatings on Copper, in: K.L. Mittal, Silanes and Other Coupling Agents, $5^{\text {th }}$ ed., 2009. 


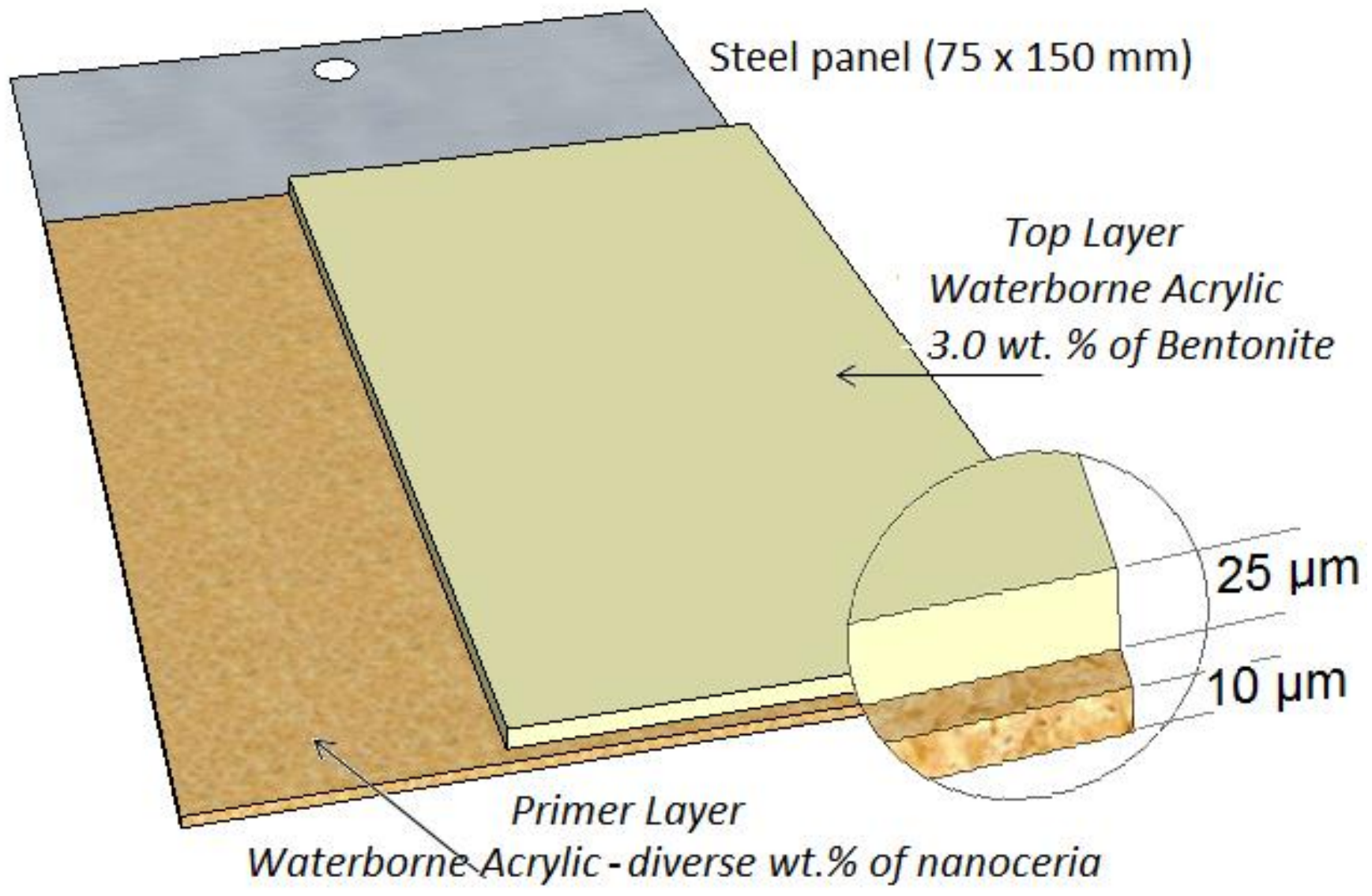

Figure 1 - Scheme of the two layer paint system 


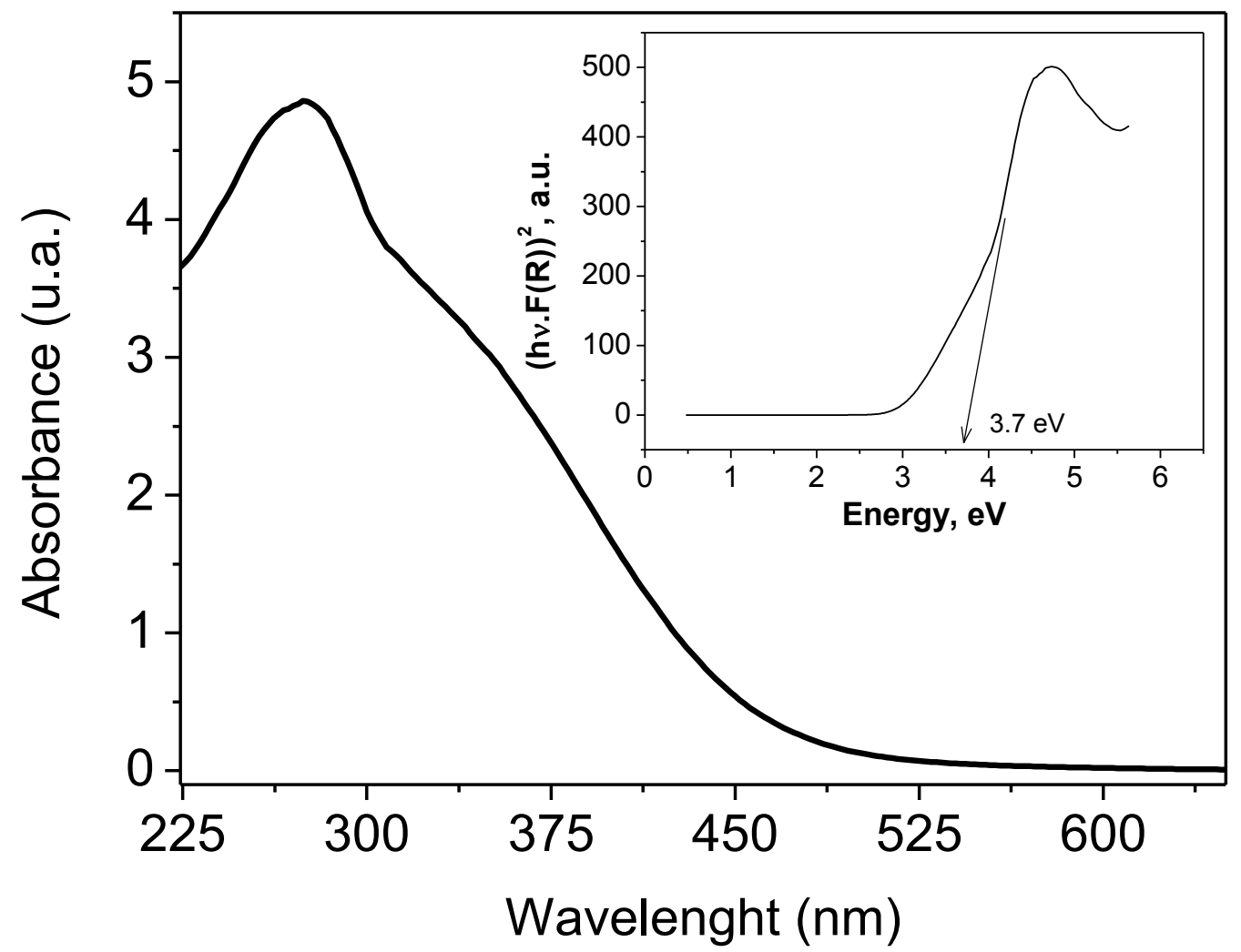

Figure 2 - UV-vis spectrum of the as-prepared $\mathrm{CeO}_{2}$ nanoparticles and the estimated band gap energy. 


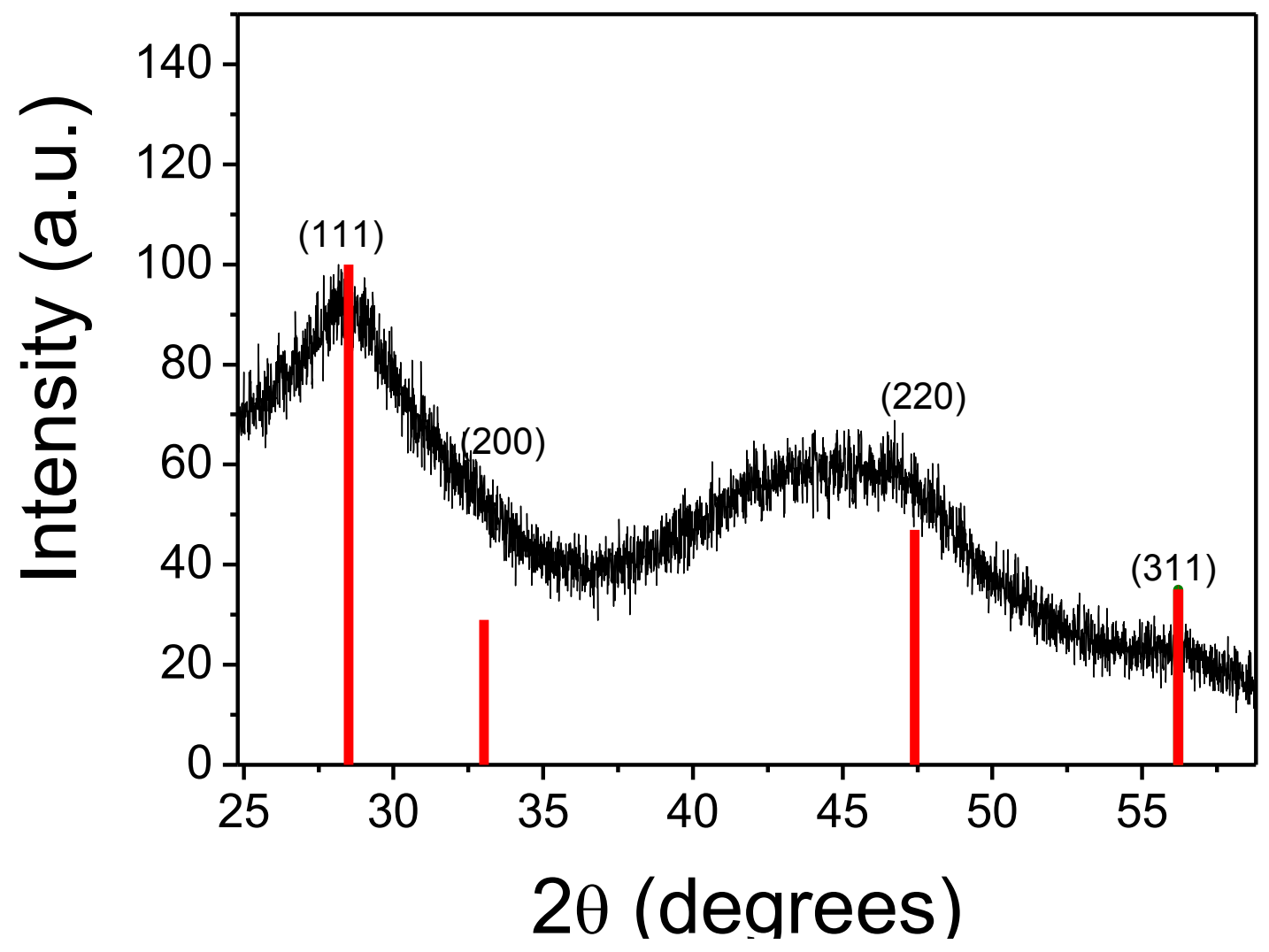

Figure 3 - XRD pattern of as-prepared $\mathrm{CeO}_{2}$ nanoparticles 


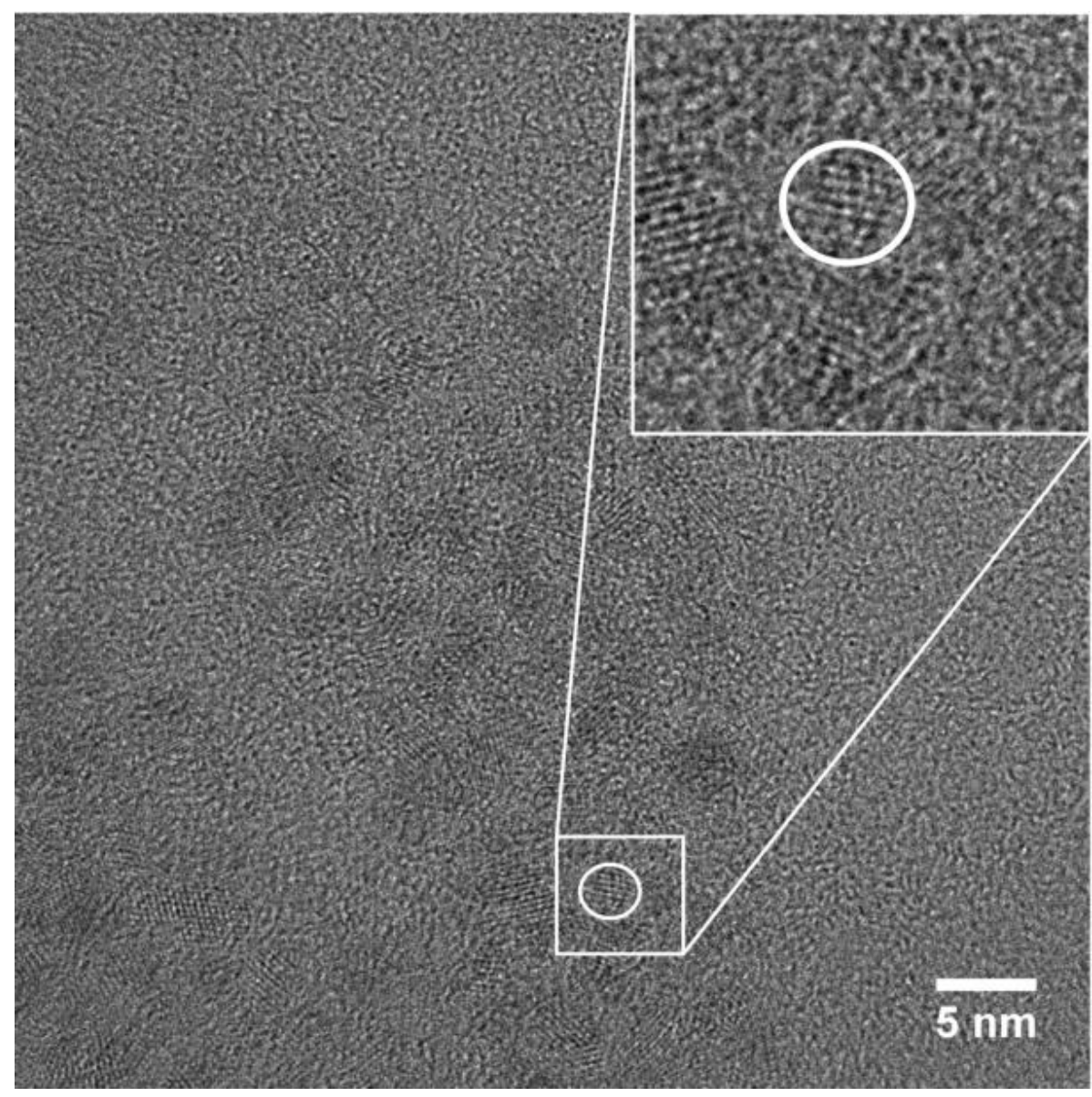

Figure 4 - High resolution TEM of $\mathrm{CeO}_{2}$ nanoparticles identifying a single crystal of nanoceria. 

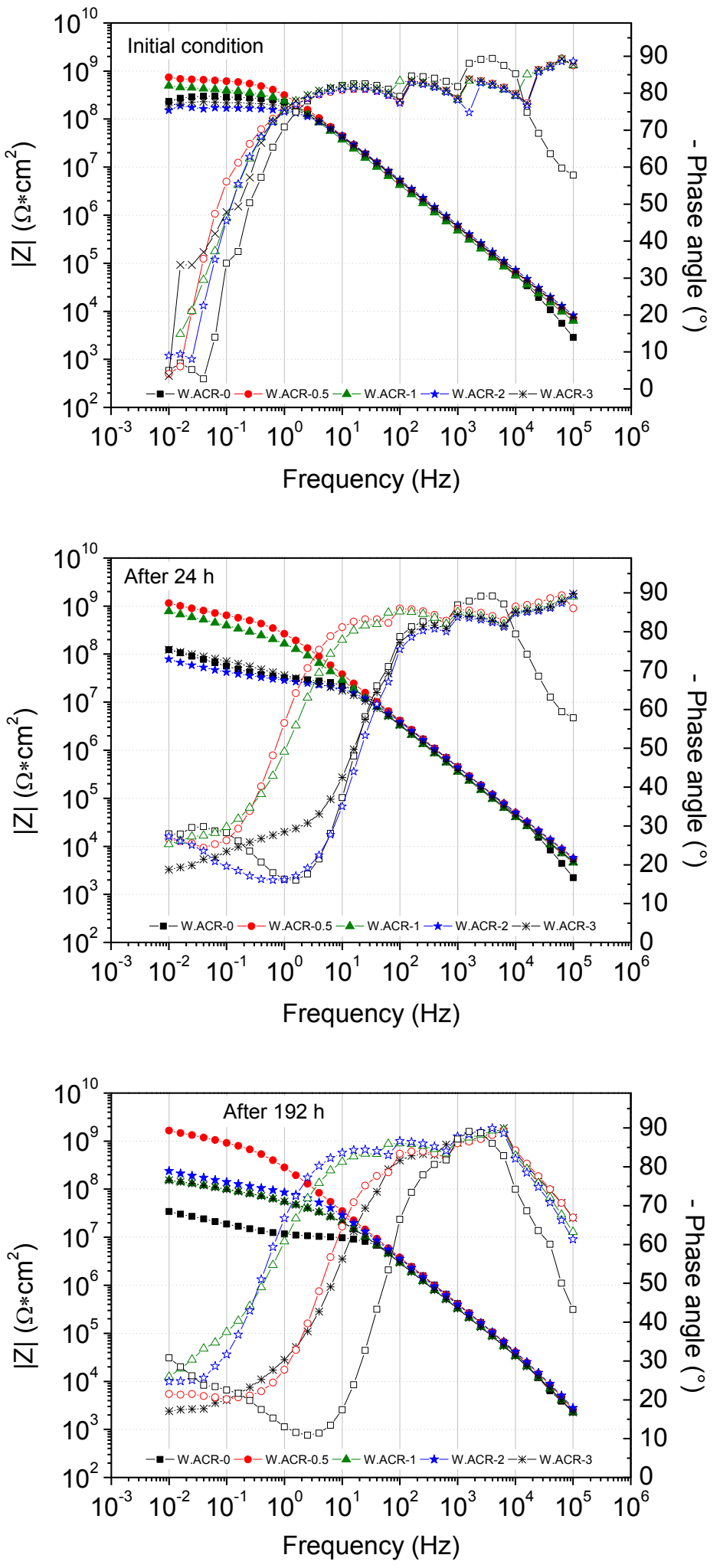

Figure 5 - EIS Bode graph of intact coatings at the beginning, after 24 and $192 \mathrm{~h}$ of exposure to sodium chloride solution 


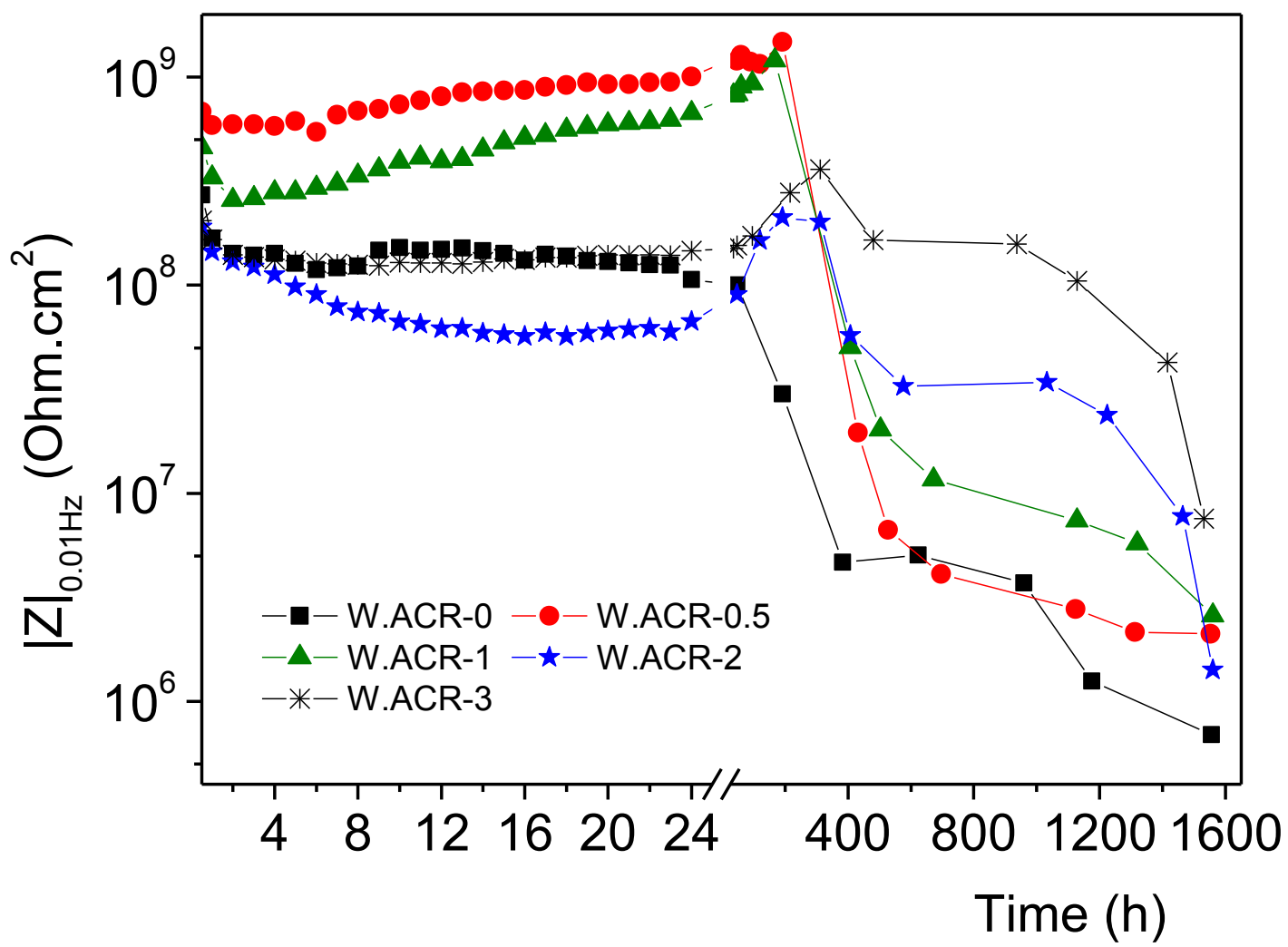

Figure 6 - Evolution of the total impedance at $0.01 \mathrm{~Hz}$ of the paint systems after $1600 \mathrm{~h}$ of exposure to the sodium chloride solution 

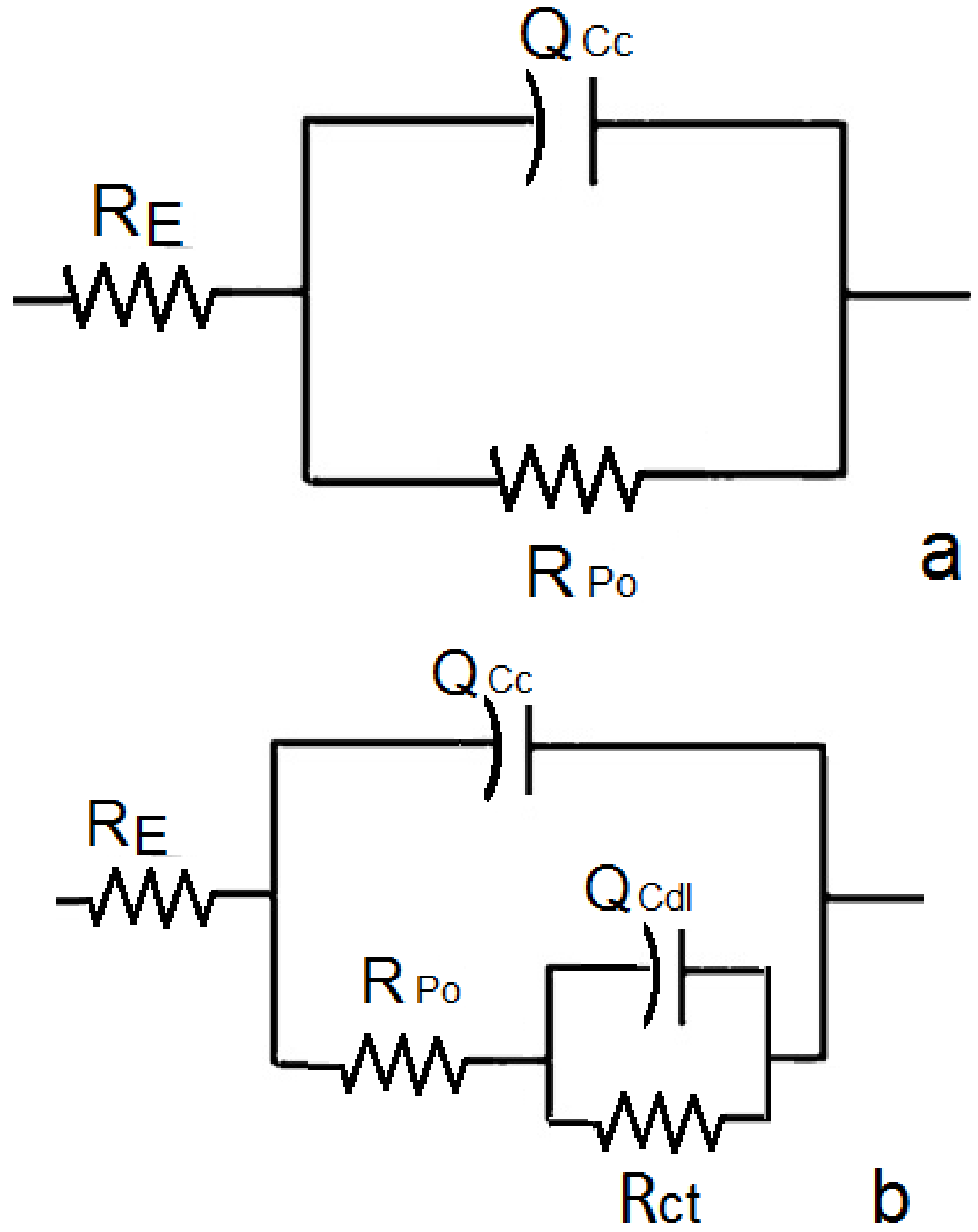

Figure 7 - Electrical circuits: (a) One-time constant $\operatorname{Re}\left(Q_{C c} . R_{P_{0}}\right)$ and (b) two-time constants $\operatorname{Re}\left(\mathrm{QCc}_{\left(\mathrm{R}_{\mathrm{Po}}\right.}\right.$ $(\mathrm{Qdl} . \mathrm{Rct})$ )). Re - electrolyte resistance; QCc - Coating capacitance; Rpo - Pore resistance; Qdl - Double layer capacitance and Rct - Charge transfer resistance. 

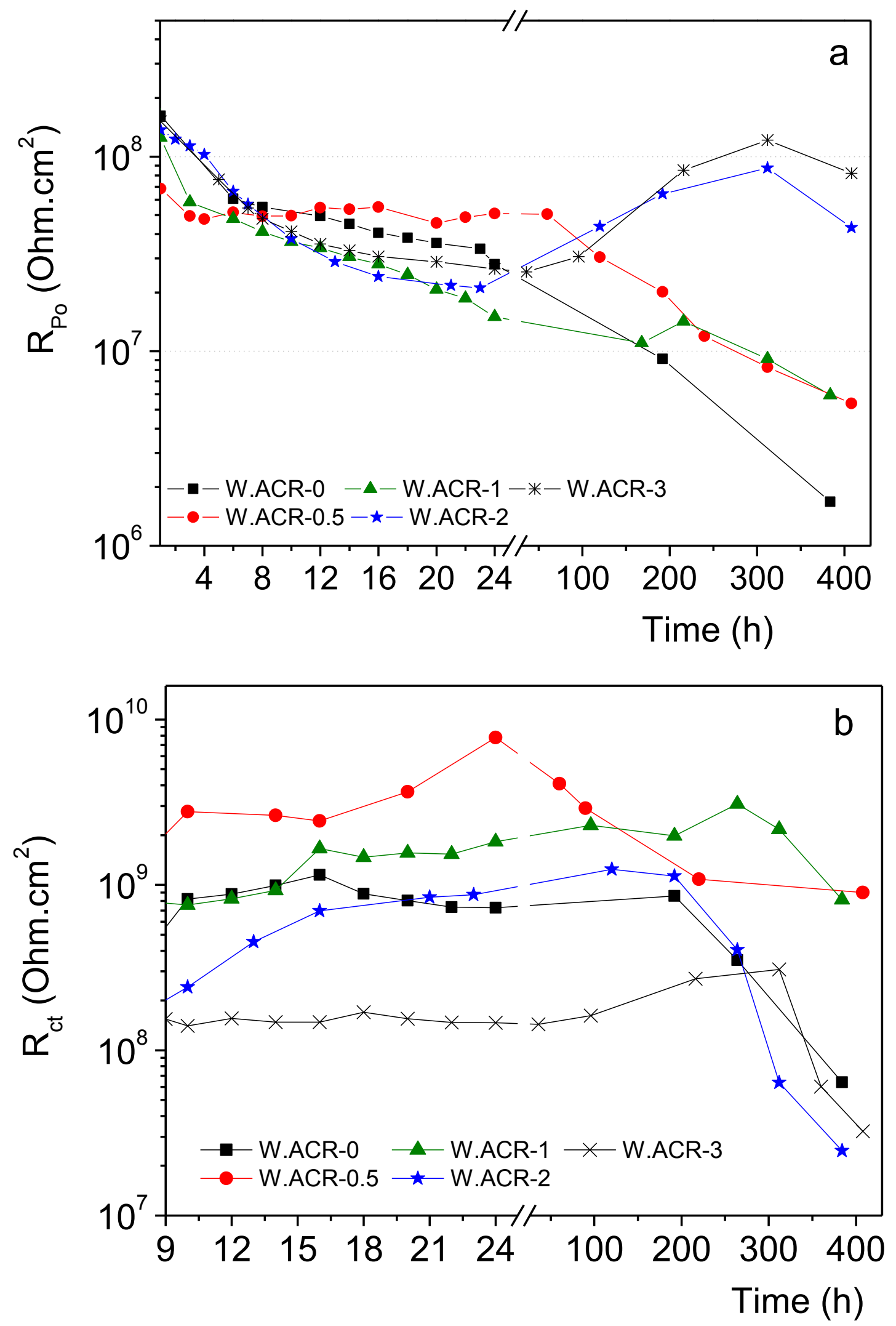

Figure 8 - Evolution of the pore resistance of paint systems after $400 \mathrm{~h}$ of exposure to sodium chloride solution. 


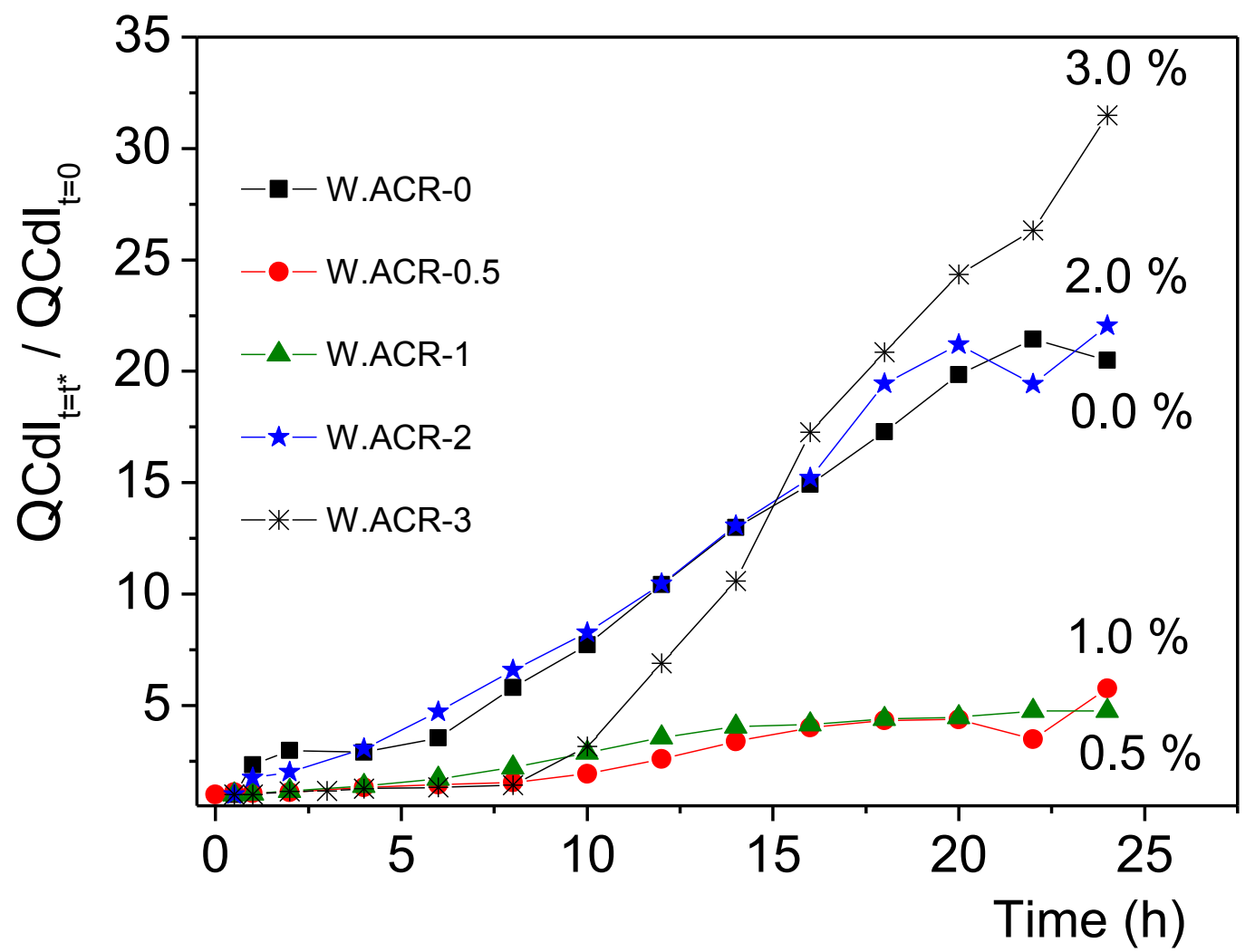

Figure 9 - Evolution of the normalized double layer capacitance for the paint systems in presence of the macroscopic defect. 


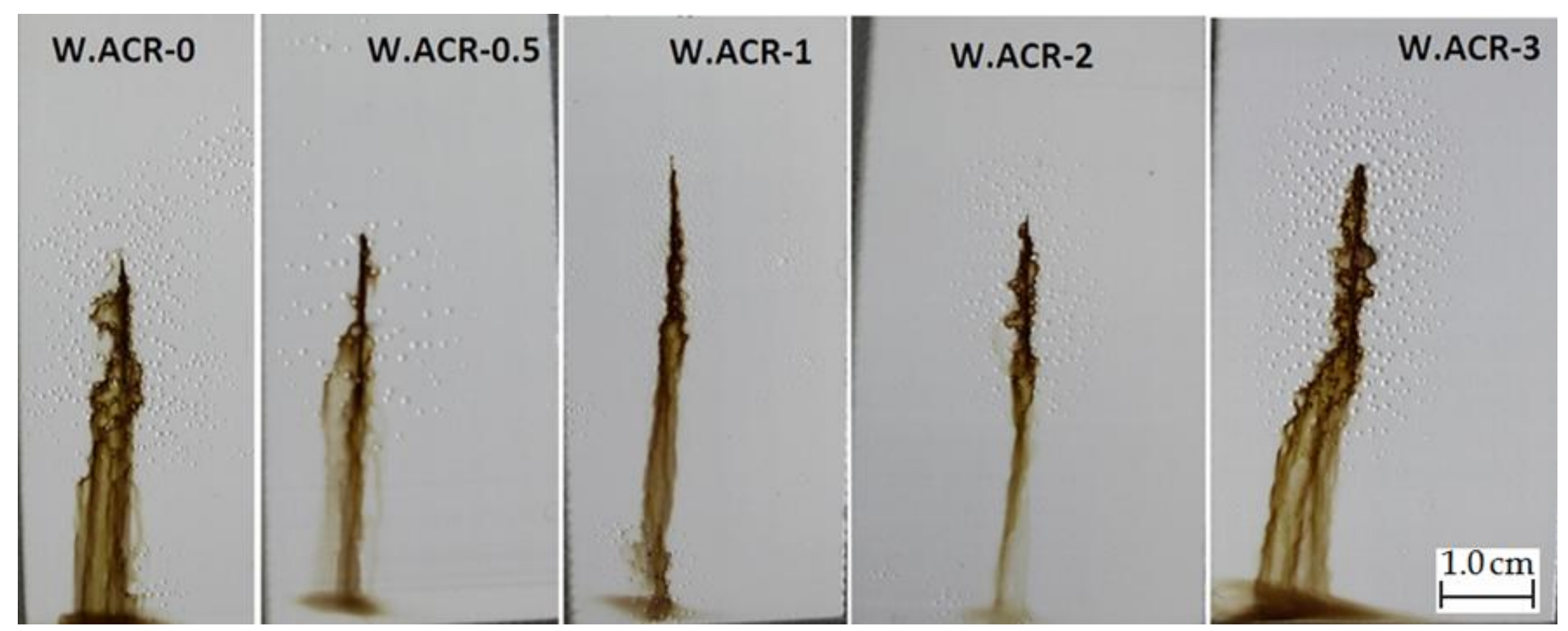

Figure 10 - Scratched panels after $24 \mathrm{~h}$ of exposure inside salt spray chamber 
Table 1 - Main features of the acrylic binder. Technical data from Arkema Coating Resins

\begin{tabular}{cc}
\hline Polymer backbone & Styrene 2 Ethyl Hexyl Acrylate \\
Solid Content [21] & $49,8 \%$ \\
Glass Transition temperature & $4{ }^{\circ} \mathrm{C}$ \\
Binder viscosity [22] & $1450 \mathrm{mPa} . \mathrm{s}$ \\
\hline
\end{tabular}


Table 2 - Main features of the coating systems

\begin{tabular}{cccc}
\hline Label & $\begin{array}{c}\text { Primer layer } \\
\mathrm{CeO}_{2}(\text { wt. } \%)\end{array}$ & $\begin{array}{c}\text { Top Layer } \\
\text { Bentonite }\end{array}$ & $\begin{array}{c}\text { Total Thickness } \\
(\mu \mathrm{m})\end{array}$ \\
\hline W_ACR-0 & 0.0 & & $34.1 \pm 2.6$ \\
W_ACR-0.5 & 0.5 & $33.6 \pm 2.4$ \\
W_ACR-1.0 & 1.0 & 3.0 wt. \% & $33.5 \pm 2.3$ \\
W_ACR-2.0 & 2.0 & & $32.9 \pm 2.8$ \\
W_ACR-3.0 & 3.0 & $33.9 \pm 2.4$ \\
\hline
\end{tabular}


Table 3 - Two conditions of the EIS investigation of the waterborne coated panels

\begin{tabular}{ccc}
\hline EIS conditions & $\begin{array}{c}\text { Signal } \\
\text { amplitude }\end{array}$ & Electrolyte solution \\
\hline Intact coatings & $20 \mathrm{mV}$ & $0.1 \mathrm{M} \mathrm{NaCl}$ \\
$1 \mathrm{~cm}$ long defect & $5 \mathrm{mV}$ & 0.3 wt. $\% \mathrm{Na}_{2} \mathrm{SO}_{4}$ \\
\hline
\end{tabular}

